\title{
ON POINTWISE ONE-SIDED ESTIMATES FOR CONTINUOUS FUNCTIONS
}

\author{
GERD HERZOG and ROLAND LEMMERT \\ Mathematisches Institut I, Universität Karlsruhe, D-76128 Karlsruhe,Germany \\ e-mail: Gerd.Herzog@math.uni-karlsruhe.de,Roland.Lemmert@math.uni-karlsruhe.de
}

In honour of Ray Redheffer

(Received 25 May, 2005; accepted 10 October, 2005)

\begin{abstract}
We prove that Mazur's functional characterization for one-sided estimates can be restricted to smaller classes of functionals in the case in which the functions under consideration are continuous. We apply this result to stability problems for dynamical systems in $l^{\infty}$, and in the Banach space of all selfadjoint operators on a Hilbert space.
\end{abstract}

2000 Mathematics Subject Classification. 47H06, 34G20.

1. Introduction. Let $(E,\|\cdot\|), E \neq\{0\}$, be a real Banach space, and let $m_{+}$: $E \times E \rightarrow \mathbb{R}$ denote the directional derivatives of the norm given by

$$
m_{+}[x, y]=\lim _{h \rightarrow 0+} \frac{\|x+h y\|-\|x\|}{h} .
$$

For a function $f: D \rightarrow E, D \subseteq E$, and a function $\alpha:[0, \infty) \rightarrow \mathbb{R}, \alpha(0)=0$, we consider one-sided estimates of the form

$$
m_{+}[y-x, f(y)-f(x)] \leq \alpha(\|y-x\|) \quad(x, y \in D),
$$

whereat $f$ is called dissipative if $\alpha=0$.

One-sided estimates are closely connected to a priori estimates, and to stability, invariance and existence results for dynamical systems in Banach spaces; see for example [1], [4], [7], [9], and in particular Martin's monograph [2] and the references given there.

Inspired by Redheffer's and Volkmann's results on the invariance of the Siegel disk [4], and Uhl's characterization of quasimonotone increasing functions [8] we will give an equivalent characterization for one-sided estimates of continuous functions which is easily applicable to a wide range of dynamical systems.

2. Pointwise dissipativity. Let us call $f: D \rightarrow E$ dissipative at $x_{0} \in D$ in case

$$
m_{+}\left[y-x_{0}, f(y)-f\left(x_{0}\right)\right] \leq 0 \quad(y \in D) .
$$

Let $\left(E^{*},\|\cdot\|\right)$ denote the topological dual of $E$, and let $B^{*}$ be the closed unit ball in $E^{*}$.

According to Mazur's results on sublinear functionals [3], (see also [2, p. 42]) the following functional characterization of $m_{+}$is valid:

$$
m_{+}[x, y]=\max \left\{\varphi(y): \varphi \in \partial B^{*}, \varphi(x)=\|x\|\right\} .
$$


Thus, dissipativity of $f: D \rightarrow E$ at $x_{0}$ is equivalent to

$$
y \in D, \varphi \in \partial B^{*}, \varphi\left(y-x_{0}\right)=\left\|y-x_{0}\right\| \Rightarrow \varphi\left(f(y)-f\left(x_{0}\right)\right) \leq 0 .
$$

Let $S$ be a subset $\partial B^{*}$. Let us say that $S$ has property $(D)$ if

$$
M:=\{x \in E: \exists \varphi \in S \cup(-S): \varphi(x)=\|x\|\}
$$

is a dense subset of $E$.

For example, $S$ has property $(D)$ if $S \cup(-S)$ is the set of all extremal points of $B^{*}$ : Each $x \in E$ generates a weak ${ }^{*}$-continuous linear functional $\Phi_{x}$ on $E^{*}$, which attains its maximal value at an extremal point $\varphi$ of $B^{*}$. But then $\|\varphi\|=1$ and

$$
\|x\|=\left\|\Phi_{x}\right\|=\Phi_{x}(\varphi)=\varphi(x),
$$

so in this case we have $M=E$.

Furthermore, if $\operatorname{conv}(S \cup(-S))=B^{*}$, an easy calculation shows $M=E$ and

$$
m_{+}[x, y]=\sup \{\varphi(y): \varphi \in S \cup(-S), \varphi(x)=\|x\|\} .
$$

In particular $\partial B^{*}$ in (2.2) can be replaced by $S \cup(-S)$ in this case. However, if $M$ is merely dense in $E$, then equivalence of (2.2) with $\partial B^{*}$ replaced by $S \cup(-S)$ and dissipativity of $f$ at $x_{0}$ is no longer valid in general (see the example in section 5), but is still valid for continuous functions.

THEOREM 1. Let $D \subseteq E$ be open, let $x_{0} \in D$, let $f: D \rightarrow E$ be continuous, and let $S$ have property $(D)$. Then, the following assertions are equivalent.

(1) $y \in D, \varphi \in S \cup(-S), \varphi\left(y-x_{0}\right)=\left\|y-x_{0}\right\| \Rightarrow \varphi\left(f(y)-f\left(x_{0}\right)\right) \leq 0$.

(2) If $v:[0, T] \rightarrow D$ with $T>0$ is any differentiable function satisfying

$$
m_{+}\left[v(t)-x_{0}, v^{\prime}(t)-f(v(t))+f\left(x_{0}\right)\right]<0(t \in[0, T]),
$$

then $\left\|v(t)-x_{0}\right\| \leq\left\|v(0)-x_{0}\right\|(t \in[0, T])$.

(3) $f$ is dissipative at $x_{0}$.

Proof. (1) $\Rightarrow(2)$. Let $v$ be as in (2), fix any $r>\left\|v(0)-x_{0}\right\|$, and set $d(t)=v(t)-x_{0}$. Assume that $\|d(t)\|<r(t \in[0, T])$ is not valid. Then there exists $t_{0} \in(0, T]$ such that

$$
\|d(t)\|<r\left(t \in\left[0, t_{0}\right)\right), \quad\left\|d\left(t_{0}\right)\right\|=r .
$$

We have

$$
m_{+}\left[d\left(t_{0}\right), d^{\prime}\left(t_{0}\right)-f\left(v\left(t_{0}\right)\right)+f\left(x_{0}\right)\right]<0,
$$

hence, in turn, there exist $h>0, t_{1} \in\left(0, t_{0}\right)$, and $\varepsilon>0$ such that

$$
\begin{gathered}
\left\|d\left(t_{0}\right)+h\left(d^{\prime}\left(t_{0}\right)-f\left(v\left(t_{0}\right)\right)+f\left(x_{0}\right)\right)\right\|-\left\|d\left(t_{0}\right)\right\|<0, \\
\left\|d\left(t_{0}\right)+h\left(\frac{d\left(t_{1}\right)-d\left(t_{0}\right)}{t_{1}-t_{0}}-f\left(v\left(t_{0}\right)\right)+f\left(x_{0}\right)\right)\right\|-\left\|d\left(t_{0}\right)\right\|<0, \\
\left\|d\left(t_{0}\right)+z+h\left(\frac{d\left(t_{1}\right)-\left(d\left(t_{0}\right)+z\right)}{t_{1}-t_{0}}-f\left(v\left(t_{0}\right)+z\right)+f\left(x_{0}\right)\right)\right\| \\
-\left\|d\left(t_{0}\right)+z\right\|<0 \quad(z \in E:\|z\| \leq \varepsilon) .
\end{gathered}
$$


Since $x \in M$ implies $\lambda x \in M(\lambda \in \mathbb{R})$, and since the homotetic projection of $\{x \in E$ : $\|x\| \geq r\}$ onto $\{x \in E:\|x\|=r\}$ is Lipschitz continuous, we find $z \in E$ such that

$$
\|z\| \leq \varepsilon, \quad d\left(t_{0}\right)+z \in M, \text { and }\left\|d\left(t_{0}\right)+z\right\|=r .
$$

Hence there exists $\varphi \in S \cup(-S)$ such that

$$
\varphi\left(d\left(t_{0}\right)+z\right)=\left\|d\left(t_{0}\right)+z\right\|=r .
$$

Since $t_{1}<t_{0}$ we have $\left\|d\left(t_{1}\right)\right\|<r$.

In the case where $\varphi\left(d\left(t_{0}\right)+z\right)=\varphi\left(v\left(t_{0}\right)+z-x_{0}\right)=r$, keeping in mind that $\|\varphi\|=$ 1 , inequality (2.3) leads to

$$
\varphi\left(\frac{d\left(t_{1}\right)-\left(d\left(t_{0}\right)+z\right)}{t_{1}-t_{0}}-f\left(v\left(t_{0}\right)+z\right)+f\left(x_{0}\right)\right)<0 .
$$

Therefore

$$
0<\varphi\left(\frac{d\left(t_{1}\right)-\left(d\left(t_{0}\right)+z\right)}{t_{1}-t_{0}}\right)<\varphi\left(f\left(v\left(t_{0}\right)+z\right)-f\left(x_{0}\right)\right) \leq 0,
$$

a contradiction. Thus, $\|d(t)\|<r(t \in[0, T])$, and $r \rightarrow\left\|v(0)-x_{0}\right\|$ from the right proves

$$
\left\|v(t)-x_{0}\right\| \leq\left\|v(0)-x_{0}\right\| \quad(t \in[0, T]) .
$$

$(2) \Rightarrow(3)$. Let $y \in D$, and without loss of generality let $y \neq x_{0}$. Fix $\varepsilon>0$ and set

$$
v(t)=y+t\left(f(y)-f\left(x_{0}\right)-\varepsilon\left(y-x_{0}\right)\right) .
$$

First, restrict $t$ to an interval $[0, T]$ such that $v(t) \in D(t \in[0, T])$, and

$$
\begin{aligned}
\|f(y)-f(v(t))\| & -\varepsilon\left\|v(t)-x_{0}\right\|+t \varepsilon\left\|f(y)-f\left(x_{0}\right)-\varepsilon\left(y-x_{0}\right)\right\| \\
& \leq-\frac{\varepsilon}{2}\left\|y-x_{0}\right\| \quad(t \in[0, T]),
\end{aligned}
$$

which is possible since the left hand side in this inequality tends to $-\varepsilon\left\|y-x_{0}\right\|$ as $t \rightarrow 0+$.

Consider

$$
m_{+}\left[v(t)-x_{0}, v^{\prime}(t)-f(v(t))+f\left(x_{0}\right)\right]=m_{+}\left[v(t)-x_{0}, f(y)-f(v(t))-\varepsilon\left(y-x_{0}\right)\right] .
$$

Fix $t \in[0, T]$, and let $\varphi \in E^{*}$ be such that

$$
\|\varphi\|=1, \quad \varphi\left(v(t)-x_{0}\right)=\left\|v(t)-x_{0}\right\| .
$$

Then

$$
\begin{aligned}
\varphi\left(f(y)-f(v(t))-\varepsilon\left(y-x_{0}\right)\right)= & \varphi(f(y)-f(v(t)))-\varepsilon \varphi\left(v(t)-x_{0}\right)+t \varepsilon \varphi\left(f(y)-f\left(x_{0}\right)\right. \\
& \left.-\varepsilon\left(y-x_{0}\right)\right) \\
\leq & \|f(y)-f(v(t))\|-\varepsilon\left\|v(t)-x_{0}\right\|+t \varepsilon \| f(y)-f\left(x_{0}\right) \\
& -\varepsilon\left(y-x_{0}\right) \| \\
\leq & -\frac{\varepsilon}{2}\left\|y-x_{0}\right\|,
\end{aligned}
$$


so by means of (2.1)

$$
m_{+}\left[v(t)-x_{0}, v^{\prime}(t)-f(v(t))+f\left(x_{0}\right)\right] \leq-\frac{\varepsilon}{2}\left\|y-x_{0}\right\|<0 \quad(t \in[0, T]) .
$$

Consequently,

$$
\left\|v(t)-x_{0}\right\| \leq\left\|v(0)-x_{0}\right\|=\left\|y-x_{0}\right\| \quad(t \in[0, T]) .
$$

We obtain

$$
m_{+}\left[y-x_{0}, f(y)-f\left(x_{0}\right)-\varepsilon\left(y-x_{0}\right)\right]=\lim _{t \rightarrow 0+} \frac{\left\|v(t)-x_{0}\right\|-\left\|y-x_{0}\right\|}{t} \leq 0,
$$

and, since $m_{+}$is continuous in its second variable, $\varepsilon \rightarrow 0+$ proves

$$
m_{+}\left[y-x_{0}, f(y)-f\left(x_{0}\right)\right] \leq 0 .
$$

(3) $\Rightarrow$ (1) follows immediately from (2.2).

3. One-sided estimates. In the sequel let $\alpha:[0, \infty) \rightarrow \mathbb{R}$ be continuous with $\alpha(0)=0$.

TheOrem 2. Let $D \subseteq E$ be open, $x_{0} \in D$, let $f: D \rightarrow E$ be continuous, and let $S$ have property $(D)$. Then, the following assertions are equivalent:

(1) $y \in D, \varphi \in S \cup(-S), \varphi\left(y-x_{0}\right)=\left\|y-x_{0}\right\| \Rightarrow \varphi\left(f(y)-f\left(x_{0}\right)\right) \leq \alpha\left(\left\|y-x_{0}\right\|\right)$;

(2) $m_{+}\left[y-x_{0}, f(y)-f\left(x_{0}\right)\right] \leq \alpha\left(\left\|y-x_{0}\right\|\right) \quad(y \in D)$.

As an immediate consequence of Theorem 2 we have a global version of this result. Note that then $S \cup(-S)$ can be replaced by $S$, since $x$ and $y$ can be interchanged.

THEOREM 3. Let $D \subseteq$ E be open, let $f: D \rightarrow$ E be continuous, and let $S$ have property (D). Then, the following assertions are equivalent:

(1) $x, y \in D, \varphi \in S, \varphi(y-x)=\|y-x\| \Rightarrow \varphi(f(y)-f(x)) \leq \alpha(\|y-x\|)$;

(2) $m_{+}[y-x, f(y)-f(x)] \leq \alpha(\|y-x\|) \quad(x, y \in D)$.

Proof of Theorem 2. (1) $\Rightarrow$ (2). Let $g: D \rightarrow E$ be defined by

$$
g(y)=\frac{\alpha\left(\left\|y-x_{0}\right\|\right)}{\left\|y-x_{0}\right\|}\left(y-x_{0}\right) \quad\left(y \in D \backslash\left\{x_{0}\right\}\right), \quad g\left(x_{0}\right)=0,
$$

and note that $g$ is continuous everywhere since $\alpha(0)=0$. Let

$$
y \in D, \varphi \in S \cup(-S), \varphi\left(y-x_{0}\right)=\left\|y-x_{0}\right\| .
$$

Then

$$
\varphi\left(f(y)-g(y)-\left(f\left(x_{0}\right)-g\left(x_{0}\right)\right)\right)=\varphi\left(f(y)-f\left(x_{0}\right)\right)-\alpha\left(\left\|y-x_{0}\right\|\right) \leq 0 .
$$

According to Theorem 1

$$
m_{+}\left[y-x_{0}, f(y)-g(y)-\left(f\left(x_{0}\right)-g\left(x_{0}\right)\right)\right] \leq 0 \quad(y \in D),
$$


and since $m_{+}$satisfies the triangle inequality in its second variable we have

$$
m_{+}\left[y-x_{0}, f(y)-f\left(x_{0}\right)\right] \leq m_{+}\left[y-x_{0}, g(y)\right]=\alpha\left(\left\|y-x_{0}\right\|\right) \quad(y \in D) .
$$

Finally, (2) $\Rightarrow$ (1) follows from (2.1).

4. Applications to differential equations in $l^{\infty}$. Let $A$ be a nonempty set, and let $l^{\infty}(A)$ denote the Banach space of all bounded functions $x: A \rightarrow \mathbb{R}$ endowed with the supremum norm. Let

$$
S=\left\{\varphi_{a}: \varphi_{a}(x)=x_{a}, a \in A\right\} .
$$

Here $M$ is the set of all $x \in l^{\infty}(A)$ where $\left\{\left|x_{a}\right|: a \in A\right\}$ has a maximum. Obviously $S$ has property $(D)$.

Now, let $D \subseteq l^{\infty}(A)$ be open, let $f: D \rightarrow l^{\infty}(A)$ be continuous, and let $x_{0} \in D$ with $f\left(x_{0}\right)=0$. Assume that

$$
m_{+}\left[y-x_{0}, f(y)-f\left(x_{0}\right)\right] \leq-L\left\|y-x_{0}\right\| \quad(y \in D),
$$

for some $L>0$ let $g: D \rightarrow l^{\infty}(A)$ be continuous and such that for some $c>0$

$$
(g(x))_{a} \geq c \quad(x \in D, a \in A) .
$$

Within these settings we have the following result. Then

Theorem 4. Let $F: D \rightarrow l^{\infty}(A)$ be defined by $(F(x))_{a}=(g(x))_{a} \cdot(f(x))_{a}(a \in A)$.

$$
m_{+}\left[y-x_{0}, F(y)\right] \leq-c L\left\|y-x_{0}\right\| \quad(y \in D) .
$$

Proof. Fix $a \in A$ and let $y \in D$. Theorem 2 applies since $\varphi_{a}\left(y-x_{0}\right)=y_{a}-\left(x_{0}\right)_{a}=$ $\left\|y-x_{0}\right\|$ implies

$$
\begin{aligned}
\varphi_{a}\left(f(y)-f\left(x_{0}\right)\right) & =(f(y))_{a} \leq-L\left\|y-x_{0}\right\| \\
\Rightarrow \varphi_{a}\left(F(y)-F\left(x_{0}\right)\right) & =(g(y))_{a} \cdot(f(y))_{a} \leq-c L\left\|y-x_{0}\right\|,
\end{aligned}
$$

and $-\varphi_{a}\left(y-x_{0}\right)=-\left(y_{a}-\left(x_{0}\right)_{a}\right)=\left\|y-x_{0}\right\|$ implies

$$
\begin{aligned}
-\varphi_{a}\left(f(y)-f\left(x_{0}\right)\right) & =-(f(y))_{a} \leq-L\left\|y-x_{0}\right\| \\
\Rightarrow-\varphi_{a}\left(F(y)-F\left(x_{0}\right)\right) & =-(g(y))_{a} \cdot(f(y))_{a} \leq-c L\left\|y-x_{0}\right\| .
\end{aligned}
$$

For example, let $f: l^{\infty}(A) \rightarrow l^{\infty}(A)$ be continuous, and let

$$
m_{+}[y-x, f(y)-f(x)] \leq-L\|y-x\| \quad\left(x, y \in l^{\infty}(A)\right),
$$

with $L>0$. According to Martin's fixed point Theorem [2, p. 257], $f(x)=0$ has a unique solution $x_{0} \in l^{\infty}(A)$. Let $g: l^{\infty}(A) \rightarrow l^{\infty}(A)$ be continuous, and such that to each bounded set $B \subseteq l^{\infty}(A)$ there is a constant $\gamma_{B}>0$ with

$$
(g(x))_{a} \geq \gamma_{B} \quad(x \in B, a \in A) .
$$


By means of Theorem 4, for each $r>0$ there is some constant $c_{r}>0$ such that

$$
m_{+}\left[y-x_{0}, F(y)\right] \leq-c_{r} L\left\|y-x_{0}\right\| \quad\left(\left\|y-x_{0}\right\| \leq r\right) .
$$

In addition, let $f, g$ be Lipschitz continuous on bounded subsets of $l^{\infty}(A)$. Then the initial value problem

$$
v^{\prime}(t)=F(v(t)), \quad v(0)=v_{0}
$$

is uniquely locally solvable. Let $v:\left[0, \omega_{+}\right) \rightarrow l^{\infty}(A)$ be the nonextendable solution to the right, let $r=\left\|v_{0}-x_{0}\right\|$ and $\rho(t)=\left\|v(t)-x_{0}\right\|$. According to [2, p. 228],

$$
\left\|v-x_{0}\right\|_{+}^{\prime}(t)=m_{+}\left[v(t)-x_{0}, v^{\prime}(t)\right] \quad\left(t \in\left[0, \omega_{+}\right)\right),
$$

and thus

$$
\left\|v-x_{0}\right\|_{+}^{\prime}(t)=m_{+}\left[v(t)-x_{0}, F(v(t))\right] \leq-c_{\rho(t)} L\left\|v(t)-x_{0}\right\| \quad\left(t \in\left[0, \omega_{+}\right)\right) .
$$

Hence $\rho(t) \leq r\left(t \in\left[0, \omega_{+}\right)\right)$and therefore

$$
\left\|v(t)-x_{0}\right\| \leq \exp \left(-c_{r} L t\right)\left\|v_{0}-x_{0}\right\| \quad\left(t \in\left[0, \omega_{+}\right)\right) .
$$

In particular, $\omega_{+}=\infty$ and the stationary solution $x_{0}$ is globally asymptotically stable.

For example, let $A=\mathbb{Z}$, and consider $f, g: l^{\infty}(\mathbb{Z}) \rightarrow l^{\infty}(\mathbb{Z})$ defined by

$$
\begin{aligned}
& f(x)=\left(x_{n+1}-3 x_{n}+x_{n-1}\right)_{n \in \mathbb{Z}}, \\
& g(x)=\left(\frac{1+\left(x_{n}\right)^{2}}{1+\left(x_{n+1}-x_{n}\right)^{2}}\right)_{n \in \mathbb{Z}} .
\end{aligned}
$$

It is easy to check that $m_{+}[y-x, f(y)-f(x)] \leq-\|y-x\|$ on $l^{\infty}(\mathbb{Z})$. Therefore $x_{0}=0$ is a globally asymptotically stable solution of the differential equation in (4.1) which reads

$$
u_{n}^{\prime}(t)=\frac{1+\left(u_{n}(t)\right)^{2}}{1+\left(u_{n+1}(t)-u_{n}(t)\right)^{2}}\left(u_{n+1}(t)-3 u_{n}(t)+u_{n-1}(t)\right), \quad n \in \mathbb{Z} .
$$

Note that this equation can be considered as a semidiscretization of the quasilinear parabolic equation

$$
u_{t}=\frac{1+u^{2}}{1+u_{x}^{2}}\left(u_{x x}-u\right)
$$

5. A counterexample for discontinuous functions. The following example shows that the equivalence in Theorem 1 is no longer valid for discontinuous functions. Let $f: l^{\infty}(\mathbb{N}) \rightarrow l^{\infty}(\mathbb{N})$ be defined as

$$
f(x)=\left(\sin \left(n^{2}\left(\|x\|-\left|x_{n}\right|\right)\right)\right)_{n=1}^{\infty},
$$

and let $S$ be as in section 4 for $A=\mathbb{N}$. Then $\left|\varphi_{n}(y)\right|=\left|y_{n}\right|=\|y\|$ implies $\varphi_{n}(f(y))=0$. On the other hand consider

$$
z=\left(8-\frac{\pi}{2 n^{2}}-\frac{2 \pi}{n}\right)_{n=1}^{\infty}
$$


Then $\|z\|=8$ and $n^{2}\left(\|z\|-z_{n}\right)=\pi / 2+2 n \pi(n \in \mathbb{N})$. Therefore $f(z)=(1,1,1, \ldots)$ and $m_{+}[z, f(z)]=1$.

6. Applications to Operator differential equations. Let $\mathcal{H} \neq\{0\}$ be a complex Hilbert space with inner product $(\cdot, \cdot)$, and let $\mathcal{L}_{S}$ denote the real Banach space of all continuous linear selfadjoint operators on $\mathcal{H}$, endowed with the operator norm $\|\cdot\|$.

For each $\xi \in \mathcal{H}$ let $\varphi_{\xi} \in \mathcal{L}_{s}^{*}$ be the functional

$$
\varphi_{\xi}(X)=(X \xi, \xi) \quad\left(X \in \mathcal{L}_{s}\right)
$$

We have $\left\|\varphi_{\xi}\right\|=(\xi, \xi)$.

A classical result on selfadjoint operators reads

$$
\sup _{(\xi, \xi)=1}\left|\varphi_{\xi}(X)\right|=\|X\| \quad\left(X \in \mathcal{L}_{s}\right)
$$

see for example [6, 12.25]. Moreover, each point at which this supremum is attained is an eigenvector of $X$ : If $(\eta, \eta)=1$ and $\varphi_{\eta}(X)= \pm\|X\|$, then $X \eta= \pm\|X\| \eta$, respectively.

In general the supremum in (5.1) is not a maximum, the typical examples are multiplication operators in $L^{2}$ which may have no eigenvalues, but we have the following result.

THEOREM 5. The set

$$
S=\left\{\varphi_{\xi}: \xi \in \mathcal{H},(\xi, \xi)=1\right\}
$$

has property $(D)$.

Proof. Fix $X \in \mathcal{L}_{s}$, and let $\varepsilon>0$. As a consequence of the spectral theorem for selfadjoint operators there exists a finite number of pairwise orthogonal nonzero selfadjoint projections $P_{1}, \ldots, P_{n}: \mathcal{H} \rightarrow \mathcal{H}$ with $P_{1}+\cdots+P_{n}=\mathrm{id}_{\mathcal{H}}$, and real numbers $\alpha_{1}, \ldots, \alpha_{n}$ such that

$$
\|X-Q\|<\varepsilon, \quad Q:=\sum_{k=1}^{n} \alpha_{k} P_{k}
$$

(See for example [5, p. 258], or [6, 12.24].)

Let $\left|\alpha_{m}\right|=\max _{k=1, \ldots, n}\left|\alpha_{k}\right|$. For $(\xi, \xi)=1$,

$$
\left|\varphi_{\xi}(Q)\right|=\left|\left(\sum_{k=1}^{n} \alpha_{k} P_{k} \xi, \sum_{k=1}^{n} P_{k} \xi\right)\right| \leq \sum_{k=1}^{n}\left|\alpha_{k}\right|\left(P_{k} \xi, P_{k} \xi\right) \leq\left|\alpha_{m}\right| \sum_{k=1}^{n}\left(P_{k} \xi, P_{k} \xi\right)=\left|\alpha_{m}\right|
$$

Moreover, for $\eta \in P_{m}(\mathcal{H}),(\eta, \eta)=1$,

$$
\left|\varphi_{\eta}(Q)\right|=\left|\left(\alpha_{m} P_{m} \eta, P_{m} \eta\right)\right|=\left|\alpha_{m}\right| .
$$

Hence, according to (5.1),

$$
\left|\varphi_{\eta}(Q)\right|=\left|\alpha_{m}\right|=\max _{(\xi, \xi)=1}\left|\varphi_{\xi}(Q)\right|=\|Q\| .
$$


Now let $A_{1}, \ldots, A_{n}: \mathcal{H} \rightarrow \mathcal{H}$ be continuous linear operators such that $B:=$ $\sum_{k=1}^{n}\left(A_{k}+A_{k}^{*}\right) \leq-c \operatorname{id}_{\mathcal{H}}$, that is $(B \xi, \xi) \leq-c(\xi, \xi)(\xi \in \mathcal{H})$ for some $c>0$. We consider the mapping $f: \mathcal{L}_{S} \rightarrow \mathcal{L}_{s}$ defined by

$$
f(X)=\sum_{k=1}^{n}\left(X^{j_{k}} A_{k} X^{l_{k}}+X^{l_{k}} A_{k}^{*} X^{j_{k}}\right)
$$

where $j_{1}, \ldots, j_{n}, l_{1}, \ldots, l_{n} \in \mathbb{N}_{0}$ are such that

$$
j_{k}+l_{k}=N(k=1, \ldots, n)
$$

with $N \in \mathbb{N}$ odd. Under these assumptions we have the following result.

THEOREM 6. The function $f$ satisfies

$$
m_{+}[X, f(X)] \leq-c\|X\|^{N} \quad\left(X \in \mathcal{L}_{s}\right)
$$

Proof. According to Theorem 5 we may apply Theorem 2 (with $X_{0}=0$ ). Fix $X \in \mathcal{L}_{s}$.

If $\varphi_{\eta} \in S$ and $\varphi_{\eta}(X)=\|X\|$ then $X \eta=\|X\| \eta$, and so

$$
\varphi_{\eta}(f(X))=\|X\|^{N}(B \eta, \eta) \leq-c\|X\|^{N} .
$$

Analogously, if $-\varphi_{\eta} \in S$ and $-\varphi_{\eta}(X)=\|X\|$ then $X \eta=-\|X\| \eta$, and since $N$ is odd we obtain

$$
-\varphi_{\eta}(f(X))=\|X\|^{N}(B \eta, \eta) \leq-c\|X\|^{N} .
$$

The function $f$ above is Lipschitz continuous on bounded subsets of $\mathcal{L}_{s}$, hence the initial value problem

$$
U^{\prime}(t)=f(U(t)), \quad U(0)=U_{0}
$$

is uniquely locally solvable. Let $U:\left[0, \omega_{+}\right) \rightarrow \mathcal{L}_{s}$ be the nonextendable solution to the right. Again by [2, p. 228], and by means of Theorem 6

$$
\|U\|_{+}^{\prime}(t)=m_{+}[U(t), f(U(t))] \leq-c\|U(t)\|^{N} \quad\left(t \in\left[0, \omega_{+}\right)\right) .
$$

Thus, for $N=1$,

$$
\|U(t)\| \leq \exp (-c t)\left\|U_{0}\right\| \quad\left(t \in\left[0, \omega_{+}\right)\right)
$$

and, for $N \geq 3$,

$$
\|U(t)\| \leq\left(\frac{\left\|U_{0}\right\|^{N-1}}{1+(N-1) c\left\|U_{0}\right\|^{N-1} t}\right)^{1 /(N-1)} \quad\left(t \in\left[0, \omega_{+}\right)\right) .
$$

In particular $\omega_{+}=\infty$, and the differential equation in (6.1) has $X_{0}=0$ as a globally asymptotically stable solution.

ACKNOWLEDGEMENTS. The authors are grateful to the referee for several valuable comments. 


\section{REFERENCES}

1. G. Herzog and R. Lemmert, One-sided estimates for quasimonotone increasing functions. Bull. Aust. Math. Soc. 67 (2003), 383-392.

2. R. H. Martin, Nonlinear operators and differential equations in Banach spaces (Robert E. Krieger Publ. Company, Malabar, 1987). $70-84$.

3. S. Mazur, Über konvexe Mengen in linearen normierten Räumen. Stud. Math. 4 (1933),

4. R. Redheffer and P. Volkmann, Differential equations in operator algebras. I. Invariance of the Siegel disk. Math. Ann. 239 (1979), 97-110.

5. F. Riesz, and B. Sz.-Nagy, Vorlesungen über Funktionalanalysis. Hochschulbücher für Mathematik, Band 27 (VEB Deutscher Verlag der Wissenschaften, Berlin, 1973).

6. W. Rudin, Functional analysis. (McGraw-Hill. New York, 1973).

7. S. Schmidt, Zwei Existenzsätze für gewöhnliche Differentialgleichungen in Banachräumen. Comment. Math. Prace Mat. 28 (1989), 345-353.

8. R. Uhl, Ordinary differential inequalities and quasimonotonicity in ordered topological vector spaces, Proc. Amer. Math. Soc. 126 (1998), 1999-2003.

9. P. Volkmann, Cinq cours sur les équations différentielles dans les espaces de Banach, in Topological methods in differential equations and inclusions. Proc. NATO Advanced Study Institute, Montreal, 1994. NATO Adv. Sci. Inst. Ser. C. Math. Phys. Sci., 472 (Kluwer Acad. Publ., Dordrecht, 1995), 501-520. 